\title{
Análise temporal das internações hospitalares e óbitos causados por doenças do aparelho respiratório em idosos, Minas Gerais
}

Temporal analysis of hospital admission and deaths by respiratory diseases among elderly people, Minas Gerais state, Brazil

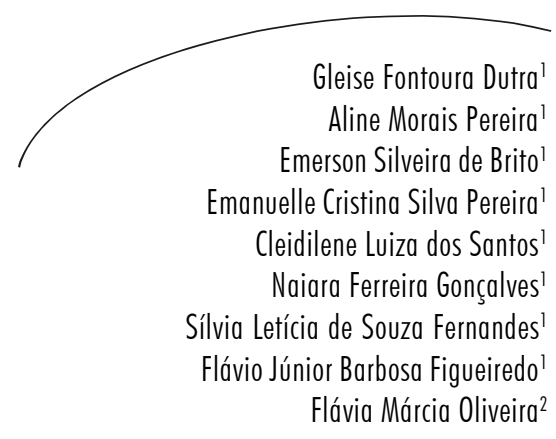

Resumo

Introdução: Os distúrbios respiratórios que acometem os idosos são condições que possuem alta taxa de morbidade e mortalidade e resultam em impactos sociais, econômicos e pessoais. Objetivo: Este estudo tem por objetivos determinar e comparar o perfil das internações hospitalares e óbitos por distúrbios respiratórios em idosos na macrorregião Leste de Saúde e na região metropolitana do Vale do Aço, numa perspectiva temporal. Métodos: Analisaram-se os dados do Ministério da Saúde referentes às internações e aos óbitos por doenças respiratórias em idosos entre 1998 a 2006. Resultados: O coeficiente de internação por doenças do aparelho respiratório em idosos na Macrorregião Leste de Saúde variou entre 2,7 a 4,3 / 100 idosos, apresentando uma redução ao longo dos anos. No entanto, na região metropolitana do Vale do Aço, a taxa de internação foi maior, apresentando uma variação entre 4,3 a 5,9 / 100 idosos. As duas principais causas de internação e óbitos em ambas as regiões correspondem às doenças obstrutivas crônicas e à pneumonia, que vêm aumentado nos últimos anos. Observou-se um efeito sazonal sobre as internações que apresentaram aumentadas nos meses de maio, junho, julho e agosto. Conclusões: Os resultados sugerem que estudos devem ser realizados de forma local, identificando as particularidades das variações dos coeficientes de morbidade e mortalidade em idosos, a fim de subsidiar políticas de saúde e determinar ações preventivas adicionais.

\section{Abstract}

Introduction: Respiratory diseases in elderly people have high morbidity and mortality levels and impacts on the social, economic and individual parameters. Objective: This study aimed to evaluate and compare in a temporal perspective the profile of hospital admissions and deaths from respiratory diseases in elderly

\footnotetext{
Centro Universitário do Leste de Minas Gerais. Ciências da Saúde. Ipatinga, MG, Brasil

2 Universidade Federal de Campina Grande. Unidade Acadêmica de Ciências da Vida. Cajazeiras, PB, Brasil

Apoio Financeiro: Bolsa de iniciação científica FAPEMIG PRT 027/2007
}

Correspondência / Correspondence

Flávia Márcia Oliveira

E-mail: fmo.75@hotmail.com

Palavras-chave: Doenças Respiratórias.

Hospitalização. Causas de Morte. Epidemiologia Descritiva. Morbidade. Idoso. 
people in the East Region and Vale do Aço, Minas Gerais State, Brazil. Methods: Brazilian Health Ministry data regarding hospital admissions and deaths from respiratory diseases between 1998 and 2006 limited to individuals aged over 60 years were analyzed. Results: The hospital admissions rates in East Region of Minas Gerais by respiratory disease were between 2.7 to 4.3 / 100 elderly people. In Vale do Aço, rates ranged from 4.3 to 5.9 / elderly people. Chronic obstructive respiratory disease and pneumonia were the major causes for hospital admission and deaths in both regions. The rate of pneumonia was increasing in the last years. A seasonal influence on hospital admissions was found in the months of May, June, July and August. Conclusions: These data suggest that studies should be conducted in small regions, so as to identify particularities of the profile of respiratory disease morbidity and mortality in elderly people to improve health care policy, and focus on additional preventive strategies.
Key words: Respiratory Tract Diseases. Hospitalization. Cause of Death. Epidemiology, Descriptive. Aged. Hospital admissions.

\section{INTRODUÇ̃̃OO}

A população idosa, considerada como aquela com 60 ou mais anos de idade, é o segmento populacional que mais cresce no país, uma vez que entre os anos de 1960 a 2000 passou de três para 14 milhões. ${ }^{1} \mathrm{O}$ envelhecimento populacional produz impacto direto nos serviços de saúde, visto que os agravos à saúde nos idosos são condições crônicas. ${ }^{1-3}$

Os distúrbios respiratórios que acometem os idosos geralmente são condições progressivamente debilitantes, apresentando alta morbidade e mortalidade, múltiplos sintomas e frequentes exacerbações que podem interferir na qualidade de vida dos indivíduos, pois geram sensação de dispneia, ansiedade, depressão, intolerância ao exercício e alteração do estado nutricional. ${ }^{4,5}$

A duração do período de morbidade desses distúrbios tem implicações pessoais, sociais e médicas importantes. Para o indivíduo, tal período representa sofrimento físico, psicológico e dificuldades financeiras. ${ }^{6}$ Observa-se, para a sociedade, um impacto com os altos custos com os serviços de saúde. ${ }^{7}$ A falta de serviços domiciliares ou ambulatoriais, bem como a falta de conhecimento sobre os fatores intrínsecos e extrínsecos que agravam as doenças respiratórias, faz com que o primeiro atendimento ocorra num estágio avançado, geralmente no âmbito hospitalar, o que aumenta os custos e diminui a probabilidade de um prognóstico favorável.
Várias estratégias têm sido adotadas para reduzir os impactos das doenças do aparelho respiratório em idosos, entre elas a vacinação contra a influenza e pneumococo. A influenza é uma doença viral aguda do trato respiratório que, nos idosos, pode levar a complicações como pneumonia e maiores taxas de hospitalização e mortalidade. Por isso, a administração dessa vacina em idosos constitui parte da política nacional. Em algumas regiões do Brasil, também é administrada a vacina contra pneumococo em idosos institucionalizados, com patologias crônicas ou imunossuprimidos. ${ }^{8}$

O objetivo do presente estudo consiste em determinar e comparar o perfil das internações hospitalares e óbitos em idosos por distúrbios respiratórios na macrorregião Leste de Saúde e na região metropolitana do Vale do Aço, numa perspectiva temporal.

\section{MÉTODOS}

\section{Caracterização da região de estudo}

A macrorregião Leste de Saúde possui 78,6\% de população urbana e compreende as microrregiões de Caratinga, Coronel Fabriciano, Governador Valadares, Ipatinga, Mantena, Santa Maria do Suaçui e Resplendor. A mortalidade geral por causas definidas nessa região se concentra nas doenças dos aparelhos circulatório e respiratório. A região metropolitana do Vale do 
Aço está incluída na macrorregião Leste e se destaca por corresponder à segunda aglomeração urbana de Minas Gerais e constituir um forte polo industrial. Além da crescente urbanização e desenvolvimento industrial, alguns municípios $\mathrm{da}$ região apresentam grande fluxo de veículos, devido à presença de rodovias importantes como a BR381, principal ligação dos estados das regiões Sul e Sudeste com a região Nordeste do país. Portanto, essa região constitui um importante local de estudo para avaliar o impacto das condições atmosféricas na taxa de internação e óbitos em função de distúrbios respiratórios.

\section{Amostragem}

Usuários do Sistema Único de Saúde (SUS) com 60 anos ou mais e cadastrados no sistema de internações hospitalares (SIH) foram selecionados para fazer parte do estudo, devido à maior susceptibilidade às doenças respiratórias nessa faixa etária. Foi realizado um levantamento do número de internações hospitalares e óbitos em idosos por diferentes doenças relacionadas ao aparelho respiratório, segundo a Classificação Internacional das Doenças - 10 (CID-10) capítulo II, ressaltando as mais significativas. Para isso, utilizaram-se as informações de saúde - epidemiológicas e morbidade hospitalar - do banco de dados do Ministério da Saúde DATASUS (http://w3.datasus.gov.br/datasus/ datasus.php), as quais foram delimitadas em espaço (macrorregião de saúde Leste de Minas Gerais e região metropolitana do Vale do Aço) e tempo (período de 1998 a 2006). Os dados foram organizados em tabelas e gráficos, para posterior análise descritiva e cálculo dos coeficientes através do programa GraphPad Prism versão 5.0.

\section{RESULTADOS}

O número de idosos na Macrorregião Leste de Saúde de Minas Gerais representou de 8,3\% a 9,17\% da população total, entre os anos de 1998 a 2007, segundo o censo e as projeções intercensitárias. Na região metropolitana do Vale do Aço, a representação dos idosos é menor, correspondendo a 5,8\% a 6,8\% entre os anos de 1998 a 2007 (figura 1). Essa diferença se deve à maior representatividade $\mathrm{da}$ população economicamente ativa na região, devido à concentração de polos industriais. Desta forma, o índice de crescimento da população idosa nas regiões de estudo, entre os anos de 1998 a 2007, correspondeu a 1,1 e 1,4 , respectivamente. 

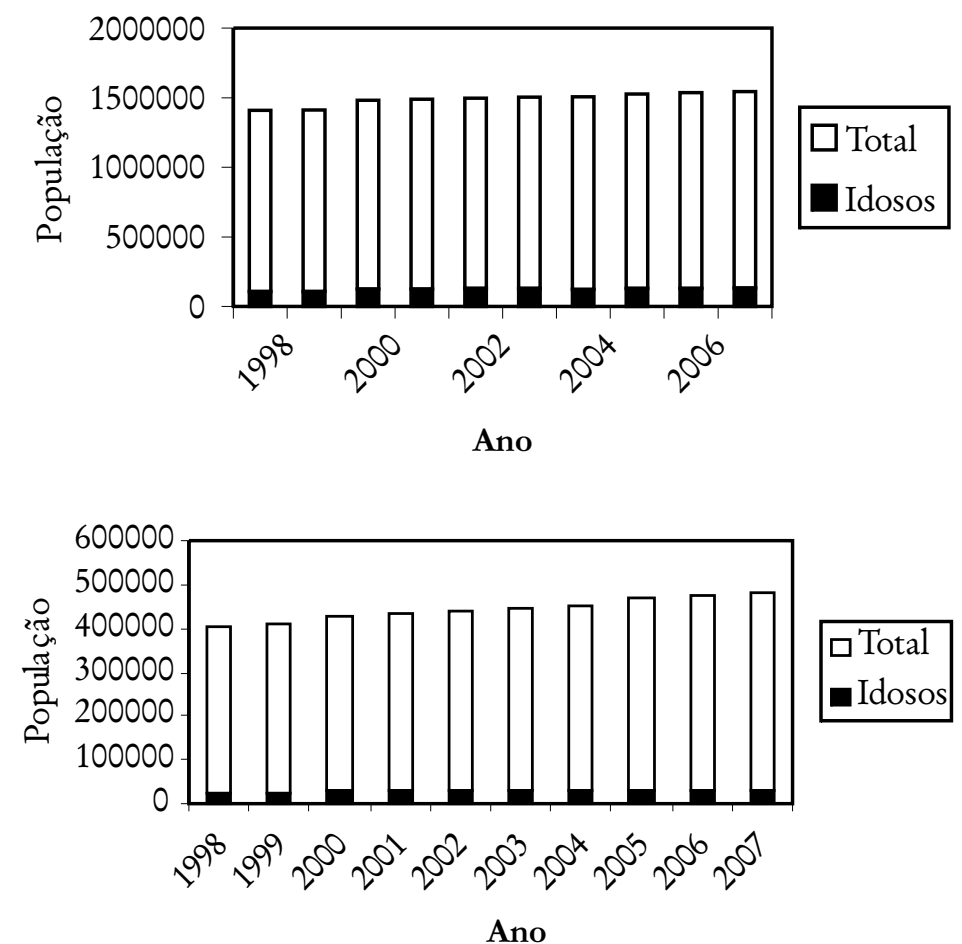

Figura 1 - Representação da população total e idosa da Macrorregião Leste de Saúde de Minas Gerais e da Região Metropolitana do Vale do Aço, respectivamente, segundo o censo e projeções intercensitárias.

O coeficiente de internação por doenças do aparelho respiratório em idosos (capítulo X / CID-10) na Macrorregião Leste de Saúde variou entre 2,7 a 4,3 / 100 idosos, apresentando redução ao longo dos anos. O mesmo perfil pode ser observado em relação ao número absoluto das internações (figura 2). No entanto, na região metropolitana do Vale do Aço, o coeficiente de internação foi maior, apresentando variação de 4,3 a 5,9 / 100 idosos. De maneira similar ao perfil do número absoluto de internações, não é possível identificar uma tendência ao longo dos anos (figura 2).

Ocoeficiente de mortalidade de idosos, decorrente de doenças respiratórias, variou entre 0,3 e 0,4 / 100 idosos na Macrorregião Leste, sem alterações significativas ao longo dos anos. No entanto, no Vale do Aço, a taxa variou entre 0,2 a 0,8 / 100 idosos com tendência ao aumento nos últimos cinco anos. A figura 2 mostra a análise temporal de óbitos por doenças do aparelho respiratório em idosos. 


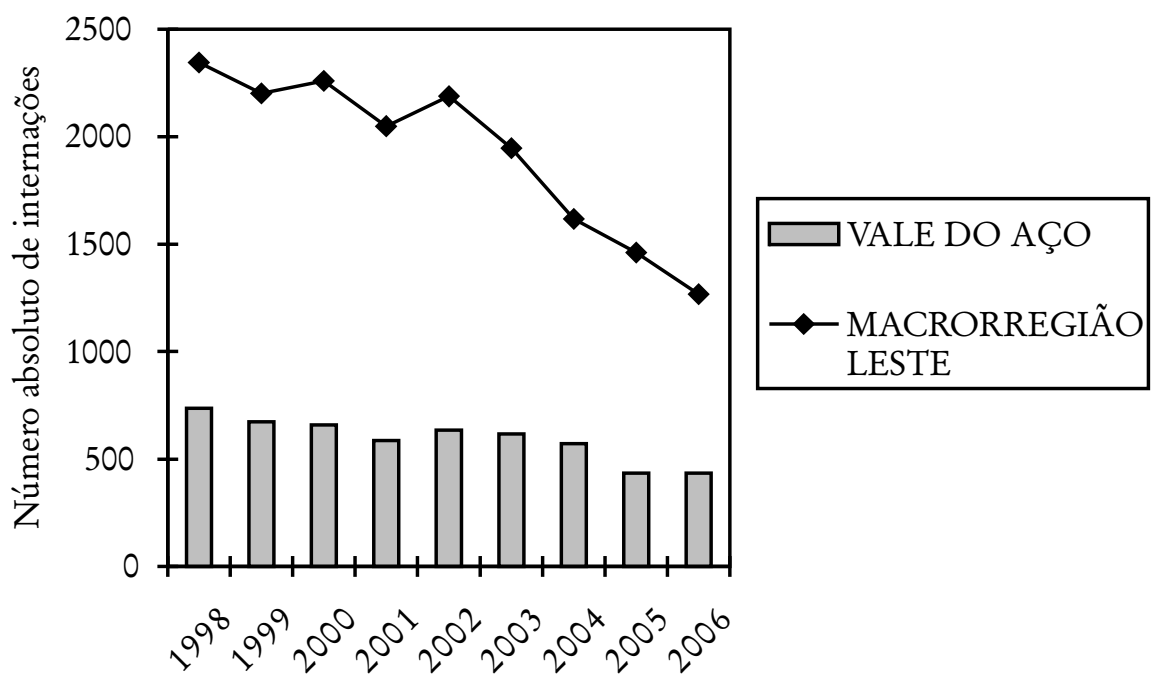

Ano

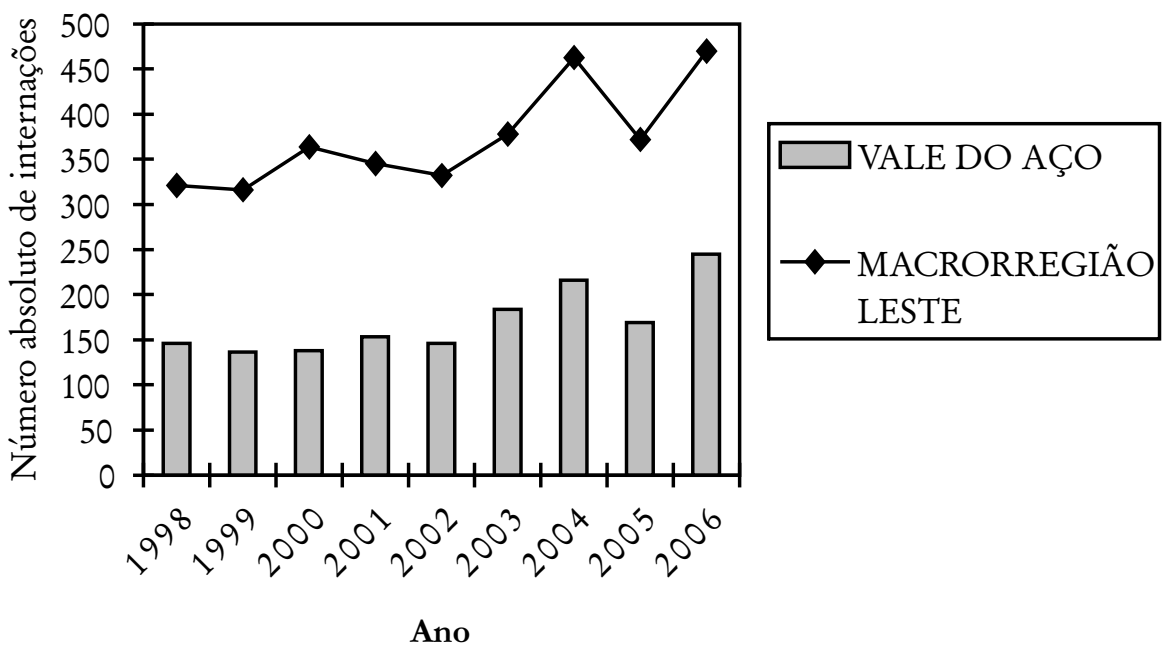

Figura 2-Número absoluto de internações e óbitos, registrado no sistema de informações de saúde (DATA-SUS), por doenças do aparelho respiratório em idosos.

De acordo com a lista de morbidade das doenças do aparelho respiratório, as doenças pulmonares obstrutivas crônicas (enfisema e bronquite) são as principais causas de internações em idosos. No entanto, o número de internações por essas doenças vem declinando progressivamente, tanto na macrorregião leste quanto no Vale do Aço no (figura 3), atingindo um índice de 0,5 de queda no ano de 2006 em relação ao ano de 1998.

A pneumonia também constitui importante causa de internação na Macrorregião Leste e no Vale do Aço, uma vez que a taxa de internação é elevada e vem apresentando oscilações ao longo dos anos (figura 3). 

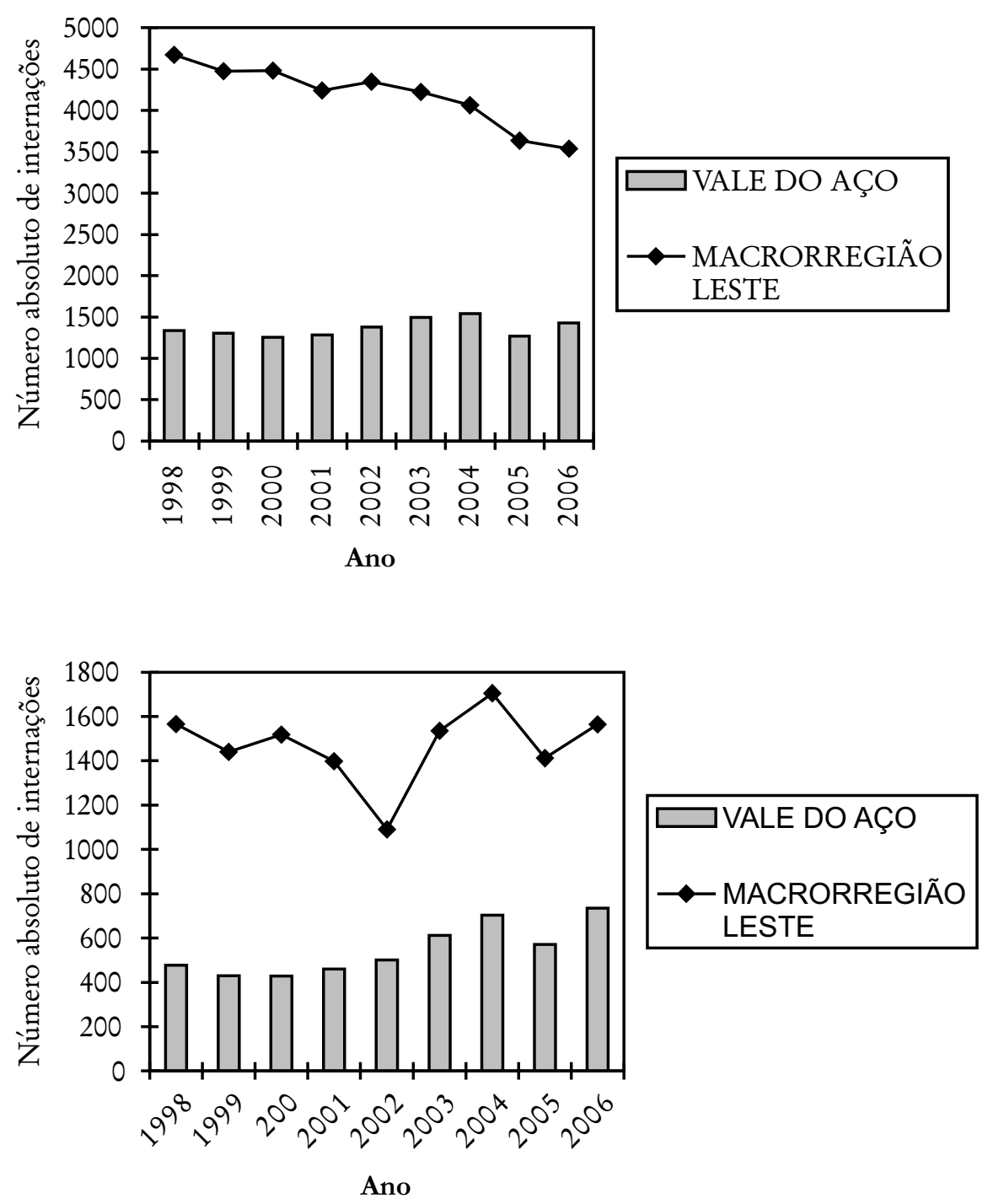

Figura 3 - Número absoluto de internações, registrado no sistema de informações de saúde (DATASUS), por doenças pulmonares obstrutivas crônicas e pneumonia, respectivamente, em idosos.

De maneira similar às internações, as doenças obstrutivas crônicas e a pneumonia são as principais causas de morte em idosos por doenças do aparelho respiratório. O perfil de mortalidade das doenças obstrutivas não seguiu um padrão específico ao longo dos anos e a pneumonia vem aumentando do número de mortes em idosos desde $2002 \mathrm{em}$ ambas as regiões de estudo (figura 4). 

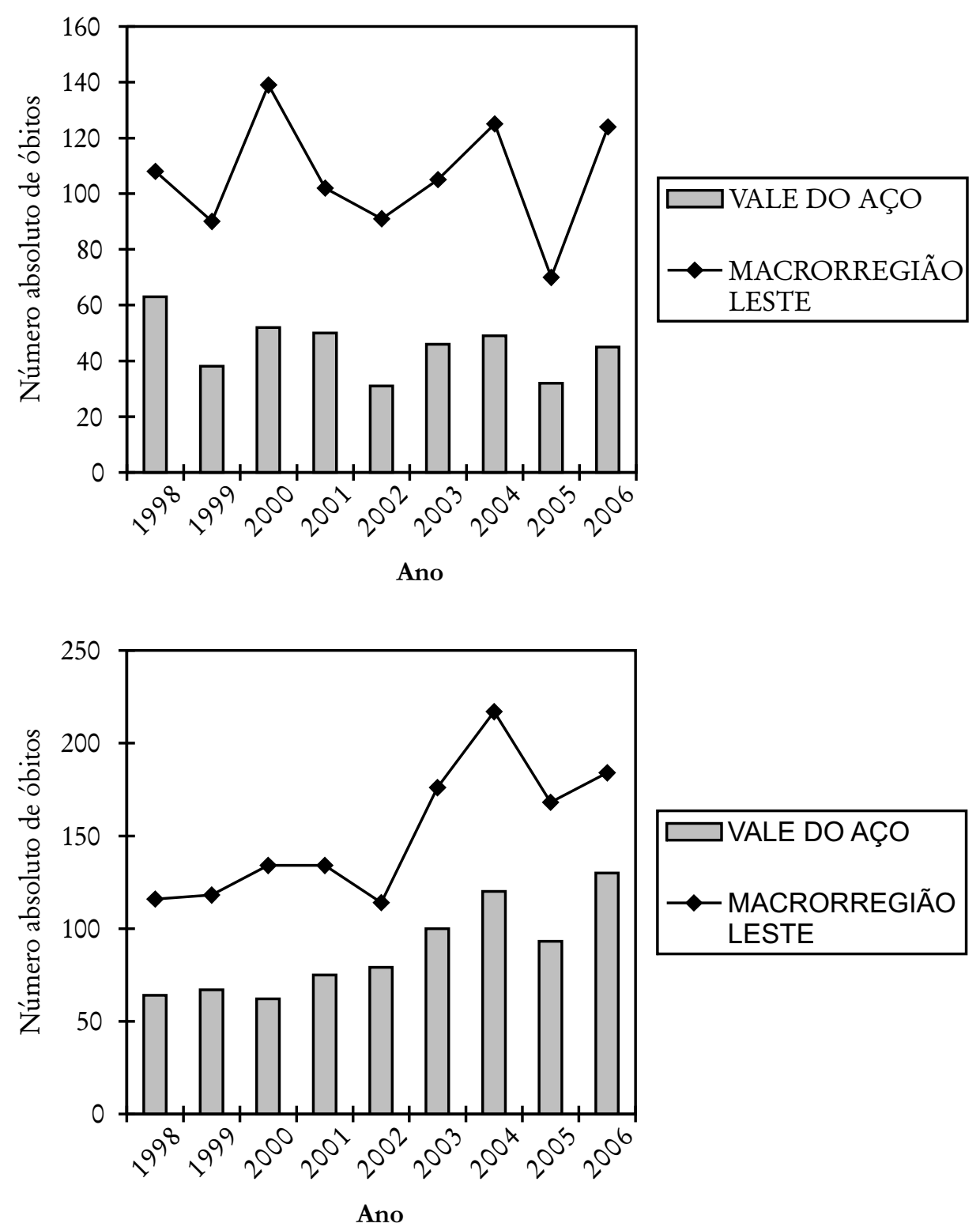

Figura 4 - Número absoluto de óbitos, registrado no sistema de informações de saúde (DATA-SUS), por doenças pulmonares obstrutivas crônicas em idosos e pneumonia em idosos, respectivamente.

Obtendo-se o coeficiente de letalidade segundo o número de óbitos / número de internações de idosos por doenças respiratórias, observa-se que a letalidade por essas doenças variou de 6,8 a 13,2 na macrorregião e de 10,9 a 17,1 no Vale do Aço.

Através da análise sazonal acumulada, foi possível determinar que as internações por doenças respiratórias como a pneumonia e doenças pulmonares obstrutivas crônicas acontecem especialmente nos meses de maio, junho, julho e agosto em ambas as regiões (tabela 1). Por outro lado, o efeito da influência sazonal sobre o número de óbitos na região metropolitana do Vale do Aço é menor (tabela 2). 
Tabela 1 - Média do número de internações por doenças respiratórias em idosos, segundo os meses dos anos de 1998 a 2006, Macrorregião Leste e Vale do Aço, Minas Gerais, Brasil.

\begin{tabular}{|c|c|c|c|c|c|c|c|c|c|c|c|c|c|}
\hline $\begin{array}{l}\text { Internação } \\
\text { macrorregião }\end{array}$ & Jan & & Fev & $\mathrm{Mar}$ & $\mathrm{Ab}$ & Mai & Jun & Jul & Ago & Set & Out & Nov & Dez \\
\hline $\begin{array}{l}\text { Capítulo X CID-10 / } \\
\text { Doenças do Aparelho } \\
\text { Respiratório }\end{array}$ & & 8,6 & 226,2 & 235 & 5,0 & 262 & 303 & 310,8 & 308,1 & 276,8 & 271,3 & 243,2 & 233,0 \\
\hline Pneumonia & & ,16 & 75,25 & 68, & 66 &, 5 & 110 & 118,9 & 113,5 & 101 & 91,83 & 79,83 & 75,66 \\
\hline $\begin{array}{l}\text { Doenças pulmonares } \\
\text { obstrutivas crônicas }\end{array}$ & & & 107,3 & 110 & 10 & 118 & 138 & 138,9 & 138,4 & 122,2 & 124,1 & 117 & 112,9 \\
\hline Asma & & 6,3 & 12,58 & 13 , & 11, & 13, & 18 & 17,66 & 15,91 & 16,33 & 17,16 & 13,08 & 17,83 \\
\hline $\begin{array}{l}\text { Outras doenças do } \\
\text { aparelho respiratório }\end{array}$ & & ,25 & 25,66 & & 21, & 66 & & 27,75 & 26,5 & 26,16 & 24,91 & 24,58 & 22,75 \\
\hline $\begin{array}{l}\text { Internação vale do } \\
\text { aço }\end{array}$ & Jan & $\mathrm{Fev}$ & $\mathrm{M}_{2}$ & & $\mathrm{Abr}$ & Maio & Jun & $\mathrm{Jul}$ & Ago & Set & Out & Nov & Dez \\
\hline $\begin{array}{l}\text { Capítulo X CID- } \\
10 \text { / Doenças do } \\
\text { Aparelho } \\
\text { Respiratório }\end{array}$ & 73,58 & 75,5 & & 5,9 & 71 & 85,58 & 97,58 & 102,1 & 106,7 & 92,25 & 84,58 & 85,91 & 74,16 \\
\hline Pneumonia & 29,66 & 26,83 & 27 &, 83 & 30,75 & 35,66 & 35,25 & 43,16 & 44,66 & 39,25 & 34 & 31,08 & 24,08 \\
\hline $\begin{array}{l}\text { Doenças } \\
\text { pulmonares } \\
\text { obstrutivas } \\
\text { crônicas }\end{array}$ & 30,33 & 35,5 & & 4,25 & 28,83 & 36,25 & 41,66 & 42,75 & 44,25 & 39,41 & 35,5 & 28,91 & 35,91 \\
\hline Asma & 4,08 & 3,75 & & 3,83 & 3,83 & 4,08 & 5,83 & 4,33 & 5,33 & 4,41 & 4,91 & 4,66 & 4,66 \\
\hline $\begin{array}{l}\text { Outras doenças do } \\
\text { aparelho } \\
\text { respiratório }\end{array}$ & 6,16 & 7,83 & & 7,33 & 6,33 & 6,75 & 7,75 & 9,91 & 8,75 & 8 & 8,41 & 8,5 & 7,08 \\
\hline
\end{tabular}


Tabela 2 - Média do número de óbitos por doenças respiratórias em idosos, segundo os meses dos anos de 1998 a 2006, Região Metropolitana do Vale do Aço, Minas Gerais, Brasil.

\begin{tabular}{|c|c|c|c|c|c|c|c|c|c|c|c|c|}
\hline Óbitos - macrorregião & Jan & Fev & Mar & Abr & Maio & Jun & Jul & Ago & Set & Out & Nov & Dez \\
\hline $\begin{array}{l}\text { Capítulo X CID-10 / } \\
\text { Doenças do Aparelho } \\
\text { Respiratório }\end{array}$ & 20,08 & 22 & 20 & 24,25 & 27,02 & 26,08 & 28,58 & 23 & 25,41 & 24,58 & 20,25 & 18,75 \\
\hline Pneumonia & 7,75 & 7,6 & 9,6 & 7 & 9,83 & 11,58 & 8,83 & 12,3 & 11,41 & 10,58 & 10,25 & 7,3 \\
\hline $\begin{array}{l}\text { Doenças pulmonares } \\
\text { obstrutivas crônicas }\end{array}$ & 5,66 & 7,33 & 5,25 & 5,16 & 6,75 & 7,66 & 7,66 & 7,58 & 6 & 7,16 & 7,16 & 6,58 \\
\hline Asma & 1,5 & 1,5 & 1 & 1,5 & 3 & 1,3 & 3 & 3 & 1 & 1 & 0 & 5 \\
\hline $\begin{array}{l}\text { Outras doenças do } \\
\text { aparelho respiratório }\end{array}$ & 6,08 & 5,91 & 4,75 & 5,25 & 7,08 & 6,75 & 7,08 & 6,83 & 5,5 & 7 & 6,41 & 5,66 \\
\hline
\end{tabular}

\begin{tabular}{|c|c|c|c|c|c|c|c|c|c|c|c|c|}
\hline Óbitos - vale do aço & Jan & $\mathrm{Fev}$ & Mar & $\mathrm{Abr}$ & Maio & Jun & Jul & Ago & Set & Out & Nov & Dez \\
\hline $\begin{array}{l}\text { Capítulo X CID-10 / } \\
\text { Doenças do Aparelho } \\
\text { Respiratório }\end{array}$ & 9,16 & 10,66 & 9,6 & 7,5 & 11,8 & 10,75 & 11,5 & 13,75 & 11,41 & 10,83 & 11 & 8,91 \\
\hline Pneumonia & 4,25 & 5,75 & 5,75 & 4,41 & 5,83 & 5,75 & 4,91 & 6,5 & 6,41 & 5,41 & 5,66 & 3,16 \\
\hline $\begin{array}{l}\text { Doenças pulmonares } \\
\text { obstrutivas crônicas }\end{array}$ & 2,58 & 3,08 & 2,16 & 2,33 & 3 & 2,75 & 3,25 & 3,25 & 3 & 2,75 & 3,41 & 2,33 \\
\hline Asma & 0,25 & 0,83 & 0 & 0,83 & 0 & 0,16 & 0,83 & 0,33 & 0 & 0 & 0 & 0,16 \\
\hline $\begin{array}{l}\text { Outras doenças do } \\
\text { aparelho respiratório }\end{array}$ & 2 & 1,58 & 1,75 & 1,25 & 2 & 2,08 & 3 & 2,16 & 1,66 & 2,66 & 2,33 & 2,58 \\
\hline
\end{tabular}

\section{DISCUSSÃO}

No Brasil, nas últimas décadas, vem-se ampliando o interesse em utilizar bancos de dados para informações em saúde como ferramenta na elaboração de políticas, no planejamento e gestão de serviços. ${ }^{9}$ Sendo assim, o Ministério da Saúde (MS), em conjunto com outros setores, criou o Departamento de Informática do Sistema Único de Saúde (DATASUS), segundo a portaria do Ministério da Saúde $\mathrm{n}^{\circ}$ 130/GM, de 12 de fevereiro de 1999. O DATASUS tem a responsabilidade de coletar, processar e disseminar as informações de saúde em âmbito nacional, ${ }^{10}$ com o objetivo de suporte técnico e administrativo às Secretarias Estaduais e Municipais de Saúde.

O presente estudo, através da análise temporal, demonstrou as similaridades e diferenças entre os perfis de internações, óbitos e letalidade de uma região metropolitana e a sua respectiva macrorregião de saúde. Tal fato reforça a importância da utilização desses sistemas de informações em saúde para subsidiar ações municipais, regionais e estaduais.

A utilização das informações disponíveis nos diversos sistemas de informação de saúde pode ser útil não apenas para o mapeamento de natalidade, diagnóstico de morbidade, mas também para o monitoramento contínuo e prospectivo. Possibilita-se, então, a criação de um sistema de alerta ou de vigilância que permita, quando ativado, desencadear intervenções terapêuticas ou preventivas para evitar a ocorrência do óbito. ${ }^{11}$

Segundo os resultados obtidos neste estudo, foi possível verificar que a região metropolitana do Vale do Aço apresentou taxas piores de morbidade, mortalidade e letalidade por doenças do aparelho respiratório em idosos, quando comparadas às da Macrorregião Leste de Saúde. Além disso, observou-se aumento expressivo das mesmas, nos últimos dois anos, especialmente em 2006. Portanto, a pneumonia ainda constitui o 
principal problema na região estudada e os fatores envolvidos no aumento do número de internações e óbitos devem ser investigados.

De modo similar, estudos nacionais e internacionais vêm demonstrando resultados similares. Pesquisa realizada em São Paulo com o objetivo de determinar o impacto da cobertura vacinal contra a influenza em idosos também demonstrou aumento expressivo da mortalidade por doenças do aparelho respiratório a partir de 2002, apesar do aumento da cobertura. ${ }^{12} \mathrm{Na}$ Bahia também foi demonstrada tendência crescente da morbidade por pneumonia em toda população nos últimos três anos. ${ }^{13}$ No Ceará, o aumento do número de internações por pneumonia na população idosa também foi descrito mesmo após a vacina para influenza. ${ }^{14} \mathrm{Em}$ Portugal, no ano de 2006, também houve aumento representativo da pneumonia como a principal causa de morte por distúrbios respiratórios e quarta causa de morte geral. ${ }^{15}$ Apesar da importância das estratégias de imunização de idosos, pesquisa recente demonstrou que a vacina contra o influenza não modifica o padrão das internações emergenciais em idosos, especialmente no inverno, sugerindo a necessidade de ações preventivas adicionais nesse período. ${ }^{16}$

Ao se investigar a distribuição das doenças respiratórias na população idosa, torna-se fundamental considerar a cobertura da vacinação contra o influenza e pneumococo, a capacidade do desenvolvimento da resposta imune individual, o perfil de circulação dos microrganismos respiratórios e os fatores ambientais.

A taxa de cobertura vacinal ainda é bastante heterogênea em diversas regiões, devido a fatores sociais, culturais, econômicos e demográficos. Estudos demonstram que a etnia e a presença de polipatologias e de equipes de saúde da família no local de residência interferem no padrão da cobertura vacinal. ${ }^{17,18}$ Além disso, em vários países foram verificados a circulação precoce do vírus da influenza - antes do início da campanha vacinal ${ }^{19,20}$ e o aumento da incidência de outros microrganismos que afetam o aparelho respiratório. ${ }^{19,21}$ Tais fatores que também podem justificar o aumento das internações e óbitos por doenças respiratórias em idosos.

O padrão da resposta imune em idosos induzido pela vacina contra o pneumococo e influenza ainda não está bem estabelecido. No entanto, alguns estudos sugerem uma taxa de efetividade entre $48 \%$ e $81 \%$ na prevenção de doenças respiratórias. ${ }^{22-24}$ Essa eficácia pode estar relacionada à heterogeneidade da resposta imune contra os antígenos vacinais. A vacina contra $o$ pneumococo, por exemplo, induz um título de anticorpos diversificado para cada sorotipo. ${ }^{25}$ Diferenças na resposta imune contra o influenza também foram descritas em estudo que demonstrou que idosos mais jovens possuíam taxas de soroconversão e proteção mais satisfatórias. ${ }^{26}$ Alguns autores também têm mostrado a correlação entre o aumento de atendimento clínico de doenças respiratórias com picos de poluição ambiental e ocorrência de baixas temperaturas. ${ }^{27-29}$.

Outro fator que deve ser considerado dentro desta perspectiva é a resistência aos antibióticos, uma vez que ainda persistem as prescrições de terapêuticas inadequadas como, por exemplo, indicação de antibióticos para as pneumonias virais e utilização de espectros não adequados. ${ }^{30,31}$

Por último, associada a essas variáveis, é importante ressaltar os problemas relacionados à qualidade da informação deste banco de dados. Embora o DATASUS seja um programa muito representativo e importante para gestores e pesquisadores da área, ainda é um sistema que possui muitas limitações. Estas ocorrem devido ao fato de não abranger todo território nacional, não possuir um claro interesse epidemiológico, ser restrita aos usuários do sistema público de saúde, possuir distorções decorrentes de alterações fraudulentas, de subnotificações, de erros no preenchimento dos prontuários ou formulários e apresentar dificuldade no acesso às informações. ${ }^{32}$ Outra limitação deste estudo consiste na ausência de dados que permitam o cruzamento de informações mais complexas e essenciais para um estudo mais profundo das causas como, por exemplo, cobertura vacinal. 


\section{CONSIDERAÇÕES FINAIS}

Os resultados reforçam a necessidade do aumento da vigilância etiológica e incorporação de indicadores ambientais para a detecção precoce e prevenção de agravos à saúde dos idosos em relação às doenças do aparelho respiratório, uma vez que o número de internações por pneumonia vem aumentando, apesar das ações preventivas utilizadas pelas equipes de saúde.

É necessária a realização de novos estudos que avaliem associações entre as diversas variáveis envolvidas nas doenças por aparelhos respiratórios, a fim de esclarecer possíveis fatores causais e contribuir para o melhor planejamento das ações preventivas de saúde e intervenções mais efetivas para os agravos.

\section{REFERÊNCIAS}

1. Lima-Costa MF, Veras R. Saúde pública e envelhecimento. Cad Saúde Pública 2003; 19: 700-1.

2. Blackman DK, Kamimoto LA, Smith SM. Overview: surveillance for selected public health indicators affecting older adults - United States. MMWR CDC Surveill Summ 1999 dec; 48(SSO8): 1-6.

3. Toyoshima MTK, Ito GM, Gouveia N. Morbidade por doenças respiratórias em pacientes hospitalizados em São Paulo/SP. Rev Assoc Med Bras 2005; 51(4): 209-13.

4. Pereira LC, Mercadante EF. Doença pulmonar obstrutiva crônica no idoso: relato de caso. Textos sobre Envelhecimento 2004; 7: 1-16.

5. Silva DMGV, et al. Qualidade de vida na perspectiva de pessoas com problemas respiratórios crônicos: a contribuição de um grupo de convivência. Rev Lat Am Enfermagem 2005 fev; 13(1): 7-14.

6. Marina CS. O processo de envelhecimento no Brasil: desafios e perspectivas. Textos sobre Envelhecimento 2005; 8(1): 1-10.

7. Litvak J. El envejecimiento de la población: un desafio que va más allá del año 2000. Bol Oficina Sanit Panam 1990 jul; 109(1): 1-5.

8. Coordenadoria de Controle de Doenças. Secretaria de Estado da Saúde de São Paulo. Campanha Nacional de Vacinação para o Idoso, 2006. BEPA 2006 abr; 3(28). Disponível em: URL: http://www.cve.saude.sp.gov.br/agencia/ bepa28_idoso.htm

9. Bittencourt SA, Camacho LAB, Leal MC. O Sistema de Informação Hospitalar e sua aplicação na saúde coletiva. Cad Saúde Pública 2006 jan; 22(1): 19-30.
10. Cavalcante MTL. Cartão nacional de saúde e centrais de regulação das ações de saúde: tendências das tecnologias de informação em saúde. [tese]. Rio de Janeiro: Escola Nacional de Saúde Pública, Fundação Oswaldo Cruz; 2003.

11. Souza $\mathrm{MH}$, et al. Sistemas de informação em saúde e monitoramento de morbidade materna grave e mortalidade materna. Revista Brasileira de Saúde Materno Infantil 2006 jun; 6(2):161-8.

12. Donalisio MR, Francisco PMSB, Latorre MRDO. Tendência da mortalidade por doenças respiratórias em idosos antes e depois das campanhas de vacinação contra influenza no Estado de São Paulo - 1980-2004. Revista Brasileira de Epidemiologia 2006; 9: 32-41.

13. Silva BMP, et al. Tendência da morbimortalidade por pneumonia na região metropolitana de Salvador- 1980 a 2004. Revista Baiana Saúde Pública 2006 dez; 30(2): 294-308.

14. Façanha MC. Impacto da vacinação de maiores de 60 anos para influenza sobre as internações e óbitos por doenças respiratórias e circulatórias em Fortaleza - CE - Brasil. J Bras Pneumol 2005 set./out; 31(5): 415-20.

15. Cienciapt.net. Pneumonia continua a ser doença grave. [Acesso em 2008 feb 22]. Disponível em: URL: http:// www.cienciapt.info/ptindex.php?option $=\mathrm{com}$ content\&task $=$ view\&id $=39060 \&$ Itemid $=260^{-}$

16. Jordan RE, et al. A case-control study of elderly patients with acute respiratory illness: effect of influenza vaccination on admission to hospital in winter 2003-2004. Vaccine 2007; 25(46): 7909-13.

17. Appel A, et al. Lack of ethnic disparities in adult immunization rates among underserved older patients in an urban public health system. Med Care 2006 nov; 44(11): 1054-8. 
18. O'Malley AS, Forrest CB. Immunization disparities in older Americans: determinants and future research needs. Am J Prev Med 2006 aug; 31(2): 150-8.

19. Barros FR, et al. O desafio da influenza: epidemiologia e organização da vigilância no Brasil. Boletim Eletrônico Epidemiológico 2004; 4(1): 1-7.

20. Perret Pérez C. Vigilância de vírus respiratórios - temporada 2004. Cuad Méd Soc 2004 sep; 44(3): 139-43.

21. Lau SK, et al. Human parainfluenza virus 4 outbreak and the role of diagnostic tests. J Clin Microbiol 2005 sep; 43(9): 4515-21.

22. Sims RV, et al. The clinical effectiveness of pneumococcal vaccine in elderly. Ann Inter Med 1988 may; 108(5): 653-7.

23. Shapiro ED, et al. The protective efficacy of polyvalent pneumococcal polysaccharide vaccine. N Engl J Med 1991 nov; 325(21): 1453-60.

24. Buttler JC, et al. Pneumococcal polysaccharide vaccine efficacy: an evaluation of current recommendations. JAMA 1993 oct; 270(15): 1826-31.

25. Valenzuela TBM, et al. Immunogenicity of a 23valent pneumococcal polysaccharide vaccine in elderly residents of a long-term care facility. Braz J Infect Dis 2007 jun; 11(3): 322-6.

26. Lino VTS. Estudo da resposta imune humoral e da ocorrência de episódios de gripe após a vacinação contra influenza em idosos. [tese].
Rio de Janeiro: Escola Nacional de Saúde Pública, Fundação Oswaldo Cruz; 2001.

27. Daumas RP, Mendonça GAS, León AP. Poluição do ar e mortalidade em idosos no município do Rio de Janeiro: análise de série temporal. Cad Saúde Pública 2004 fev; 20(1):311-19.

28. Gouveia N, et al. Hospitalizações por causas respiratórias e cardiovasculares associadas à contaminação atmosférica no Município de São Paulo, Brasil. Cad Saúde Pública 2006 dez; 22: 2669-77.

29. Martins LC, et al. Relação entre poluição atmosférica e atendimentos por infecção de vias aéreas superiores no município de São Paulo: avaliação do rodízio de veículos. Revista Brasileira de Epidemiologia 2001 nov; 4(3): 220-9.

30. Tan JS. Nonresponses and treatment failures with conventional empiric regimens in patients with community-acquired pneumonia. Infect Dis Clin North Am 2004 dec; 18(4): 883-97.

31. Niederman M. Principles of appropriate antibiotic use. Int J Antimicrob Agents 2005; 26(Suppl 3): S170-5.

32. Ferla AA, et al. Informação como suporte à gestão: desenvolvimento de parâmetros para acompanhamento do sistema de saúde a partir da análise integrada dos sistemas de informação em saúde. Relatório Analítico. Rio Grande do Sul. 13 p. [Acesso em 2008 feb 22]. Disponível em: URL: http://www.opas.org.br/ observatorio/Arquivos/Sala298.pdf 